\title{
Inhibiting PKM $\zeta$ reveals dorsal lateral and dorsal medial striatum store the different memories needed to support adaptive behavior
}

\author{
Wolfgang M. Pauli, ${ }^{1}$ Alexandra D. Clark, Heidi J. Guenther, Randall C. O’Reilly, \\ and Jerry W. Rudy \\ Department of Neuroscience and Psychology, University of Colorado at Boulder, Boulder, Colorado 80309, USA
}

\begin{abstract}
Evidence suggests that two regions of the striatum contribute differential support to instrumental response selection. The dorsomedial striatum (DMS) is thought to support expectancy-mediated actions, and the dorsolateral striatum (DLS) is thought to support habits. Currently it is unclear whether these regions store task-relevant information or just coordinate the learning and retention of these solutions by other brain regions. To address this issue, we developed a two-lever concurrent variable-interval reinforcement operant conditioning task and used it to assess the trained rat's sensitivity to contingency shifts. Consistent with the view that these two regions make different contributions to actions and habits, injecting the NMDA antagonist DL-AP5 into the DMS just prior to the shift impaired the rat's performance but enhanced performance when injected into the DLS. To determine if these regions support memory content, we first trained rats on a biased concurrent schedule (Lever 1: VI 40" and Lever 2: VI 10"). With the intent of "erasing" the memory content stored in striatum, after this training we inhibited the putative memory-maintenance protein kinase $C$ isozyme protein kinase M $\zeta$ (PKM $\zeta$ ). Infusing zeta inhibitory peptide (ZIP) into the DLS enhanced the rat's ability to adapt to the contingency shift $2 \mathrm{~d}$ later, whereas injecting it into the DMS had the opposite effect. Infusing GluR2 $3 \mathrm{zY}$ into the DMS $1 \mathrm{~h}$ before ZIP infusions prevented ZIP from impairing the rat's sensitivity to the contingency shift. These results support the hypothesis that the DMS stores information needed to support actions and the DLS stores information needed to support habits.
\end{abstract}

It is generally believed that there are two systems that contribute to acquisition and response selection in instrumental conditioning paradigms: (1) an "action" system that allows the organism to extract relationships between its behavior, outcomes, and other environmental stimuli and represents these relationships as expectancies (Tolman 1948; Dickinson 1980; Dickinson and Balleine 1993; Rudy 2008), and (2) a "stimulus-response habit" system (S-R habit) that captures consistently occurring relationships between stimuli and responses and gradually builds stimulus-response associations that do not include representations of outcomes (Thorndike 1898). The action system provides the basis for a rapid assessment of the current environment-the contingencies and outcomes associated with behavior and the initial basis for generating an adaptive response. As responses are reliably repeated in associated stimulus contexts, the S-R habit system can automate these responses (Adams and Dickinson 1981; Killcross and Coutureau 2003; Everitt and Robbins 2005). In recent years, different areas of the striatum have been identified that appear to selectively support each of these two systems (Hilário and Costa 2008; Shiflett and Balleine 2011), with the dorsomedial striatum (DMS) providing critical support for the action system, and the dorsolateral striatum (DLS) providing support for the habit system (Yin et al. 2004, 2005a,b, 2006; Ragozzino 2007).

However, there is still a debate on the critical issue of just what these regions contribute when a new problem is encountered and solved. Do these components of the striatum coordinate the learning and retention of the solution by other regions of the relevant neural system (Pasupathy and Miller 2005), or do these regions themselves also store task-relevant information

'Corresponding author.

E-mail wolfgang.pauli@colorado.edu.

Article is online at http://www.learnmem.org/cgi/doi/10.1101/lm.025148.111. (cf. Pauli et al. 2010, 2012; WM Pauli, TE Hazy, and RC O'Reilly, pers. comm.)? As Shiflett and Balleine (2011) recently put it, "Although the striatum is clearly engaged during learning and performance of goal-directed and habitual actions, this does not necessarily entail that the striatum is the long-term store of associations that underlie these behaviors."

The experiments reported here were designed to determine if the DLS and DMS learn and store information needed to support instrumental behavior. In one set of experiments, we temporarily disrupted the normal functions of these regions to confirm that they make different contributions to our behavioral task-which they did. In the second set, we determined whether these regions stored information relevant to our task. Specifically, we inhibited the putative memory-maintenance protein kinase $\mathrm{C}$ isozyme protein kinase $\mathrm{M} \zeta(\mathrm{PKM} \zeta$ ) by infusing the PKM $\zeta$ inhibitor $\zeta$ pseudosubstrate inhibitory peptide (ZIP) into the striatum (cf. Sacktor 2011). The results support the hypothesis that these striatal areas are, indeed, critical sites of learning and memory for the action and S-R habit systems, respectively. In the third set of experiments, we prevented ZIP from erasing memories by infusing GluR $2_{3 Y} 1 \mathrm{~h}$ prior to injecting ZIP. This indicates that PKM $\zeta$ maintains long-term memories in the dorsal striatum by regulating GluA2-dependent AMPA receptor trafficking. Our results then open up important new questions about what specifically these striatal areas learn in instrumental tasks and how this relates to learning taking place in other areas.

\section{Results}

DMS and DLS contribute differentially to the rats' ability to adjust to contingency shifts

To study the contribution of the DMS and DLS to behavioral adaptations to contingency changes, we trained rats to press two 
levers, each associated with the same variable interval schedule of reinforcement (VI 20"). During this phase, rats were trained so that they pressed each lever at approximately the same rate.

Theoretically, after several training sessions, the relevant task information needed to support this behavior should be captured by both the action system and the habit system. To determine if the DMS and DLS contribute differently to the now wellestablished behavior and to adjusting to new contingencies, we changed the reinforcement contingencies associated with each lever. One lever was associated with a more favorable reinforcement schedule (VI 10") than the other lever (VI 40"). If detected by the rat, this shift should result in a higher probability of pressing the more favorable lever. To impair the function of striatal neurons, we infused the competitive NMDA receptor antagonist DL-AP5 or its vehicle into either the DMS or the DLS 5 min prior to the shift session. The rationale for choosing DL-AP5 is detailed in the Materials and Methods section.

The current view-that the DMS supports the action system, whereas the DLS supports the habit system-makes two predictions: (1) Impairing the function of neurons in the DMS should impair the rat's ability to rapidly shift to the more favorable lever because the DMS would no longer be able to support the action system, and (2) in contrast, impairing the function of neurons in the DLS should facilitate the shift because this area would no longer support habitual lever-pressing according to the old contiguities. This would remove competing response tendencies that would interfere with the learning and performance required by the new contingencies.

To test our hypothesis, each rat was exposed to two 2-d test cycles. On day 1 , rats continued to be reinforced on the VI $20^{\prime \prime}$ schedule. The behavior from this session served as a baseline. On day 2, the reinforcement contingencies were shifted. Specifically, a less favorable schedule (VI $40^{\prime \prime}$ ) was assigned to the rat's preferred lever and a more favorable schedule (VI 10") was assigned to the other, less-preferred lever. Between the cycles, rats were retrained for several days on the VI $20^{\prime \prime}$ schedules. DL-AP5 was injected 5 min prior to training on day 2 of the first test cycle. The vehicle was injected on day 2 of the second cycle.

Figure 1 provides a schematic representation of the locations of the cannulae tips for rats in the DLS and DMS conditions. There were no differences among groups in overall number of leverpresses per minute during the last session before the contingencies were changed (individual group means ranged from 4.58 to 5.84; $\left.F_{(3,16)}=0.45, P=0.72\right)$, and there was no difference in the rate at which rats pressed the two levers $(t=-0.78, \mathrm{df}=19, P=0.45)$.

Figure 2 shows that the predicted results were obtained. This figure compares the performance of the groups during the second

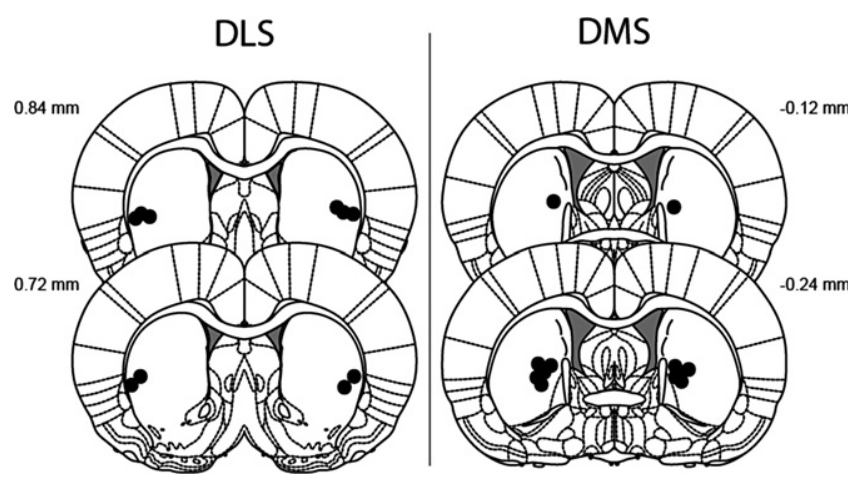

Figure 1. Schematic representation of cannulae tip placements for rats that received infusions into the DLS and DMS. Adapted from Paxinos and Watson (2008), with permission from Elsevier (C) 2008.

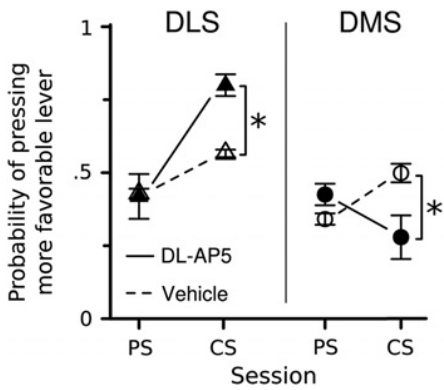

Figure 2. Probability of pressing the lever that was associated with the favorable VI 10" during the reversal session. This figure compares the performance of rats in the second half of the last session before the contingency shift to the performance during the second half of the contingency shift session. We calculated the total number of responses the rat made during the 45-min test session and then plotted the distribution of the last $50 \%$ of responses over the two levers. Rats adapted better to the contingency after infusions of DL-AP5 into the DLS than after saline vehicle infusions. In contrast, rats adapted worse to the shift after DLAP5 infusions into the DMS than after saline vehicle infusions into this striatal region. $\left(^{*}\right)$ P-value $<0.05$.

half of the preshift session to their performance during the second half of the shift session. Specifically, we calculated the total number of responses the rat made during the 45-min test session and then plotted the distribution of the last $50 \%$ of responses over the two levers. We did this on the assumption that the last half of the session would reflect the rats' adaptation to the new contingencies.

When DL-AP5 was infused into the DLS, rats adapted more readily to the contingency change than controls-they shifted more strongly to pressing the lever associated with the more favorable contingency than the other lever. In contrast, when infused into the DMS, DL-AP5 impaired the rats' ability to adapt to the contingency shift-they did not respond differentially on the two levers.

The best measure of successful adjustment to the contingency shift by an individual rat is the difference between the preshift response probability and the response probability during the shift session. Thus, for each rat we calculated the difference between the probability that the rat pressed the more favorable lever, associated with a VI-10" schedule, during the second half of the contingency shift session and the probability of pressing this lever during the second half of the preshift session. This analysis revealed that rats in the DLS drug condition adapted better to the contingency change than the rats in the DLS vehicle condition $(t=2.97, \mathrm{df}=4, P=0.02)$ and that rats in the DMS drug condition adapted worse than rats in the DMS vehicle condition $(t=-2.57, \mathrm{df}=4, P=0.03)$.

The results presented in Figure 2 are consistent with the accepted view of the contributions of the DMS and DLS. However, the results presented in Figure 3 provide a cautionary note. Figure 3 presents the average number of responses per minute made during the test session. Although there was no difference between rats in groups injected with DL-AP5 $(t=-0.38, \mathrm{df}=8, P=$ $0.72)$, overall responding of these rats was reduced compared to rats injected with saline $(t=3.51, \mathrm{df}=8, P=0.01)$. The potential problem associated with a reduced level of responding is that, compared to rats in the control condition, DL-AP5-treated rats would have reduced exposure to the new contingency, and this would reduce their ability to adjust their behavior to the contingency shift. However, if this explanation was correct, one would expect that rats in the control conditions would adapt more rapidly to the contingency shift than rats in both drug conditions. 


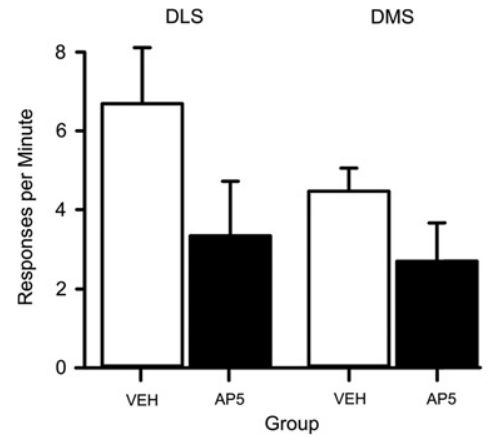

Figure 3. Number of responses per minute in the contingency-shift session. Rats pressed levers less after DL-AP5 infusions into either striatal regions than after saline vehicle infusions.

Yet, this was not the case. Even though DL-AP5-treated rats in the DLS condition responded less often than rats in the vehicle condition, they adapted to the contingency shift more quickly (as shown in Fig. 2). Thus, we don't think differences in overall response rates account for the effect of DL-AP5.

In summary, these results make three points. First, they are consistent with the hypothesis that the DLS and DMS make different contributions to the support of instrumental behavior. Impairing the DLS improved the ability of the rats to adapt to the contingency shift, presumably by removing the habit bias that would normally have to be overcome when the new contingencies were introduced. This result is consistent with Yin et al.'s (2006) report that disabling the DLS improved the rat's sensitivity to changes in reinforcement contingencies. In contrast, impairing the DMS produced the opposite effect-it reduced the rat's ability to adjust to the new contingencies, presumably because it removed access to the information needed to rapidly capture and adapt to new contingencies. This finding is consistent with the Yin et al. $(2005 \mathrm{a}, \mathrm{b})$ results and conclusions.

Second, that an infusion of DL-AP5 produced opposite effects when infused into two adjacent regions of the striatum provides the ideal control for the potential problem of site-specificity and indicates that the neurons in a particular site support the putative function. Third, these results indicate that our behavioral methodology is suitable for detecting the different contributions of the DLS and DMS to instrumental behavior and thus can be used to address the motivating question for this research-do these regions store task-relevant information?

\section{PKM $\zeta$ maintains memories in the DLS and DMS}

Do the DMS and DLS regions store information relevant to performance? Because rats in the previous experiment were tested under the influence of DL-AP5, one cannot know whether this drug blocked access to relevant memory content contained in these regions, or whether it interfered with the rat's ability to detect and adapt to the new contingencies. Ideally, to address this question one needs to have a methodology that would erase the putative memory content contained in the region of interest but leave the neurons functional at the time of the contingency shift test. Pharmacological agents that alter neuronal function during the test session or lesions methods cannot be used for this purpose.

However, an emerging literature suggests that inhibiting the function of $\mathrm{PKM} \zeta$ provides a methodology that can address this question. PKM $\zeta$ mRNA normally exists at low levels in the synaptic region but is rapidly synthesized in response to synaptic activity. A unique property of PKM $\zeta$ is that it lacks a regulatory unit, so once synthesized, it does not require second messenger binding to be in the catalytic state. In addition, once synthesized, this kinase can self-perpetuate by enabling the further translation of PKM $\zeta$ mRNA into protein to cause persistent synaptic localization (for review, see Sacktor 2011).

Two lines of evidence indicate that PKM $\zeta$ plays a special role in the maintenance of increased synaptic strength that supports memories. First, when the pseudosubstrate $\zeta$ inhibitory peptide, a molecule designed to serve as a regulatory unit for $\mathrm{PKM} \zeta$, is infused prior to the induction of long-term potential (LTP), it has no effect on induction (Serrano et al. 2005). However, if it is infused at various intervals after LTP has been induced, established potentiation is reversed, indicating that PKM $\zeta$ is critical for the maintenance of established LTP. Second, when ZIP is injected into brain regions thought to be memory storage sites, behavioral evidence for the memory is lost (Pastalkova et al. 2006; Shema et al. 2007, 2011; Serrano et al. 2008; Hardt et al. 2010; Li et al. 2011).

Important features of this class of experiments are that (1) the established memory is allowed time to consolidate before ZIP is infused into the putative storage site, (2) the animal is tested after ZIP is allowed to clear from the brain, and (3) following the memory test that reveals the memory has been lost, retraining can re-establish memory-dependent performance, indicating that the neurons supporting the memory are functional and can support new memories.

All of this suggests that PKM $\zeta$ is critical for memory maintenance and this methodology can be used as an analytic tool to determine if a particular region of the brain stores information relevant for expression of a memory-dependent behavior. Thus, we used this methodology to determine if the DLS and DMS store task-relevant information.

We reasoned that if the DMS and DLS stored task-relevant information, inhibiting PKM $\zeta$ should produce predictably different results depending on whether ZIP is infused into the DLS or DMS. Specifically, inhibition of PKM $\zeta$ in the DLS should allow rats to adapt more readily to new contingencies because it would erase the content that supports habitual behavior that normally competes with the new requirements of the task, whereas inhibiting $\mathrm{PKM} \zeta$ in DMS should interfere with adapting to the contingency shift because it would erase the content that enables the rat to assess the new contingencies against what was previously learned.

To pursue this strategy, we made a slight change in the training strategy to increase the challenge imposed by the contingency shift. During the first stage of training, the two levers were associated with the same reinforcement schedules (VI 20"). After the rats learned to press both levers, they were trained for several consecutive sessions during which one lever was associated with a more favorable VI 10" schedule and the other lever with a less favorable VI $40^{\prime \prime}$ schedule of reinforcement. During this bias training, the lever a rat had preferred during the initial acquisition was associated with the less favorable VI $40^{\prime \prime}$ and the other with the more favorable VI $10^{\prime \prime}$ schedule. Approximately $24 \mathrm{~h}$ after the last day of bias training, we either injected ZIP or an inactive scrambled version of the peptide (scr-ZIP) into the DMS or the DLS. Two d following the infusion, rats were tested by reversing the contingencies associated with each lever.

Figure 4 provides a schematic representation of the location of the injector tip locations for rats in the DLS and DMS conditions. Rats rapidly adapted to the contingencies of the bias training and pressed the lever associated with the more favorable VI $10^{\prime \prime}$ schedule more than the other lever and maintained this preference through the course of the bias training. Figure 5 shows that the predicted results were obtained when contingencies were reversed. As in the previous experiment, this figure compares the performance of the groups during the second half of the preshift session to their performance during the second half of the shift 


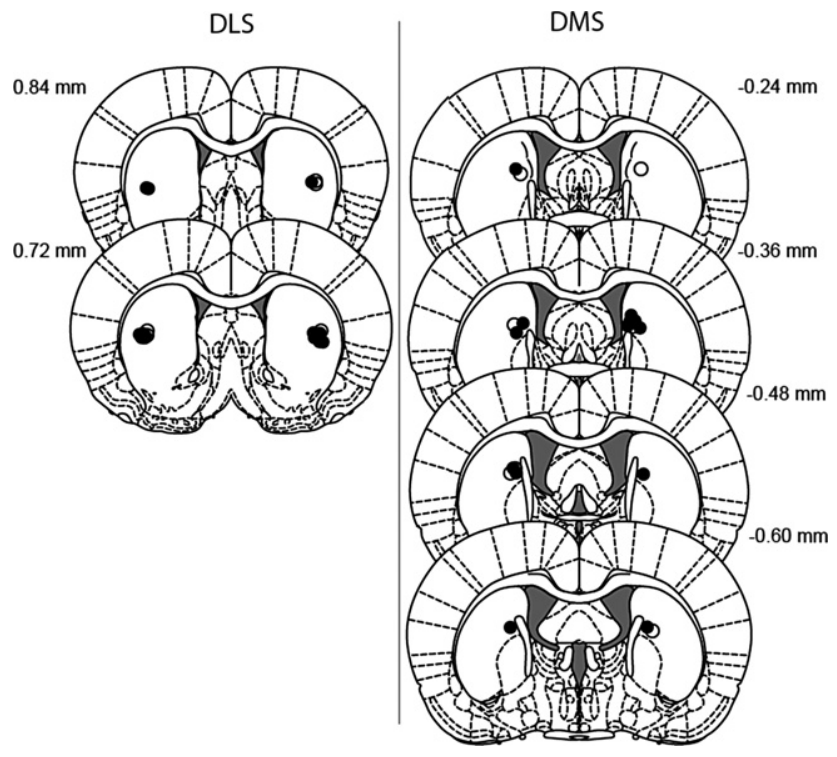

Figure 4. Schematic representation of cannulae tip placements for rats that received infusions into the DLS and DMS. Filled circles represent subjects that received ZIP infusions, open circles represent subjects that received scr-ZIP infusions. Adapted from Paxinos and Watson (2008), with permission from Elsevier (c) 2008.

session. When ZIP was infused into the DLS, rats adapted more readily to the contingency shift than controls-they pressed the lever associated with the more favorable contingency more than they pressed the other lever. In contrast, when infused into the DMS, ZIP impaired the rats' ability to adapt to the contingency shift-they did not respond differentially to two levers. As in the previous experiment, we analyzed the results by comparing preshift/post-shift difference scores. This analysis revealed that rats in the DLS drug condition adapted better to the contingency change than the rats in the DLS vehicle condition $(t=3.53, \mathrm{df}=$ $8, P=0.01)$ and that rats in the DMS drug condition adapted worse than rats in the DMS vehicle condition $(t=-2.51, \mathrm{df}=8, P=$ 0.04).

Figure 6 provides a more detailed picture of how rats adapted to the contingency reversal. In this figure, each rat's performance was normalized to the total number of responses it made during the 45-min test session. The figure plots how responses are distributed as the rat approaches its total. It shows that when ZIP was injected into the DLS, rats were already pressing the more favorable lever, even though they had only emitted $50 \%$ of their total responses. Rats in the DLS control condition, however, were still distributing their responding equally between the two levers at that point, although they did prefer the more favored lever by the end of the session. In contrast, even at the end of the session, when ZIP was injected into the DMS, rats were still distributing their responding equally between the levers.

Figure 7 shows the influence of the ZIP infusion on overall responding during the test. Compared to the src-ZIP infusion, infusion of ZIP slightly reduced overall responding $(t=-2.07, \mathrm{df}=$ $18, P=0.05)$. Note, however, that there was no difference in the two groups infused with ZIP $(t=-0.44$, df $=10, P=0.67)$. This is important because it means that the difference in the overall rate of responding is not a determiner of the influence of ZIP on the rat's ability to adapt to the new contingencies. If it was, then the DMS and DLS groups should not have differed. Yet, as shown in Figure 4, infusing ZIP into the DLS facilitated the shift to the favorable lever, but infusing it into the DMS impaired the shift.
In summary, these results support the hypothesis that the DLS and DMS store information needed for instrumental conditioning and that what was stored in the two regions had opposite effects on the rat's ability to adapt to changes in reinforcement contingencies. More specifically, the results are consistent with the idea that the DLS stores content needed to support habits and interferes with the rats' ability to rapidly adapt to changes in reinforcement contingencies, but the DMS stores content needed to rapidly assess and adapt to new contingencies.

\section{GluR2 ${ }_{3 Y}$ rescues the reversal deficit produced by dorsomedial striatal injections of ZIP}

$\mathrm{PKM} \zeta$ is thought to maintain memory by regulating trafficking of GluA2-containing AMPA receptors (AMPARs) to the spine that are needed to offset the ongoing endocytosis of these receptors. When PKM $\zeta$ is inhibited, receptors trafficked out are not replaced and the induced changes in synaptic strength are lost. This view is supported by recent experiments that have combined the infusion of ZIP with an infusion of the peptide GluR2 $2_{3 Y}$. This peptide has been shown to inhibit the endocytosis of GluA2-containing AMPARs (Migues et al. 2010; Li et al. 2011).

Given this property, GluR2 $2_{3 Y}$ provides an analytic tool to block the effect of ZIP. Specifically, if endocytosis of the GluA2containing AMPA receptors is prevented, then the temporary inactivation of PKM $\zeta$ should have no effect on memory maintenance because this renders the replacement function served by $\mathrm{PKM} \zeta$ moot-no replacement is necessary. This hypothesis has been tested and supported in experiments with both brain slices and behavior (Migues et al. 2010; Li et al. 2011). Thus, we used this strategy to determine if our results with ZIP could be related, at least indirectly, to disrupting the role of $\mathrm{PKM} \zeta$ in regulating trafficking of GluA2-containing AMPARs.

To make this assessment, we repeated one condition of the previous experiment. Approximately $24 \mathrm{~h}$ after the last bias training session, rats were assigned to two groups. Both GluR $2_{3 \mathrm{Y}}$ and ZIP were infused sequentially into the DMS of one set of rats, whereas the vehicle for GluR2 3 preceded the ZIP infusion for the other set. The GluR2 $2_{3 Y}$ infusion occurred $1 \mathrm{~h}$ prior to the ZIP infusion. If the inhibition of $\mathrm{PKM} \zeta$ erases memories by disturbing the normal trafficking of GluA2-containing AMPARs, then GluR $2_{3 Y}$ should block the effect of ZIP, and the memory content should be preserved. Given that the drugs were infused into the

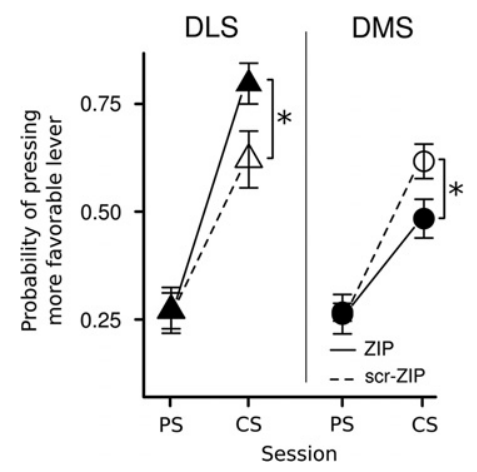

Figure 5. Probability of pressing the lever that was associated with the favorable VI 10" during the reversal session. On the day before the reversal session, when this lever was associated with the $\mathrm{VI} 40^{\prime \prime}$, rats in all groups showed a similar low probability of pressing this lever. If ZIP was infused into the DLS, rats showed an enhanced ability to adapt to the contingency change relative to control scr-ZIP injections. If ZIP was infused into the DMS, rats showed a decreased ability to adapt to the shift. Error bars indicate standard error (SE). (*) P-value $<0.05$. 


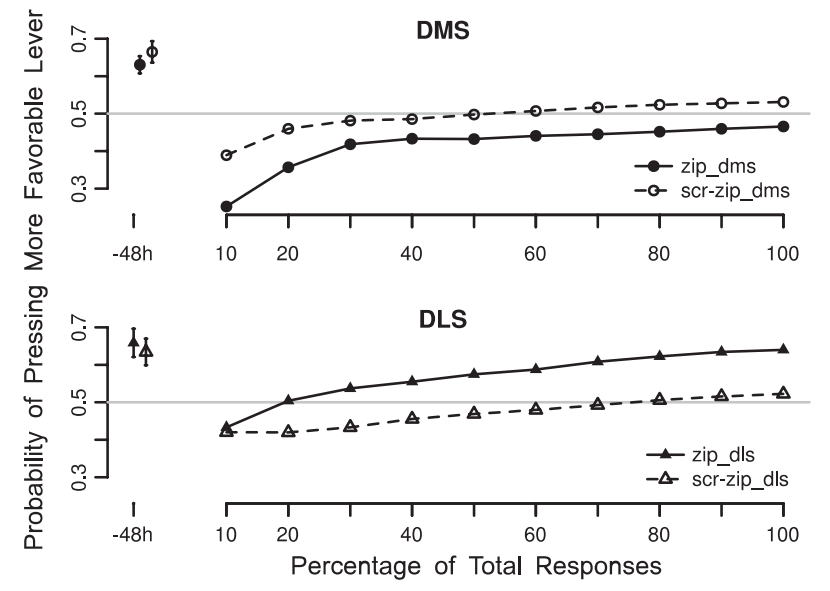

Figure 6. Adaptation to the contingency shift changes with session progress. Rats that received ZIP infusions in the DLS adapted quickly to the contingency shift by pressing the more favorable lever more frequently than the other lever and maintained this adaptation throughout the course of the session. In comparison, rats that received ZIP infusions in the DMS showed only a slow increase over time in the probability of pressing the more favorable lever more frequently than the other lever. A direct comparison of the two groups at different levels of progress through the contingency shift session indicates that the difference between the two groups appeared early in the session and persisted until the completion of the contingency shift session.

DMS, which supports the action system, this means that rats injected with both agents should adjust more rapidly to the contingency shift than rats infused with ZIP only.

Figure 8 provides a schematic representation of the injector tip locations for rats in this experiment. As in the previous experiment, the rats rapidly adapted to the contingencies and maintained this preference through the course of the bias training. During bias training, rats pressed the favorable VI-10" lever significantly more frequently $(t=6.22, \mathrm{df}=12, P=0.01)$ and received significantly more rewards from it than from the other lever $(t$-test: $t=13.53, \mathrm{df}=12, P=0.01)$.

Figure 9 presents the contingency-shift session results and reveals that the predicted results were obtained-rats infused with GluR2 $2_{3 Y}$ prior to the ZIP infusion adjusted more rapidly to the new contingencies than did rats infused with the vehicle prior to ZIP. Note that, in the case of the previous experiment, rats infused with only ZIP distributed their responses roughly equally between the two levers and thus only adapted moderately to the shift. In contrast, rats in the GluR $2_{3 y}$ conditions were more likely to press the lever associated with the more favorable VI-10" schedule. As in the previous experiment, we analyzed this interaction by comparing preshift/post-shift difference scores. This analysis revealed that rats that received infusions of GluR2 $2_{3 Y} 1 \mathrm{~h}$ before ZIP adapted better than rats that received saline before ZIP in the DMS $(t=-2.48, \mathrm{df}=11, P=0.03)$.

The differences in adapting to the new contingencies cannot be due to differences in the overall rate of responding after the contingency change, because rats in the two groups did not differ in their lever-press rate during the session $(t=-1.34, \mathrm{df}=15, P=$ 0.2 ). Rats that received GluR2 $2_{3 Y}$-ZIP pressed levers at a mean rate $17.5 \pm 2.08$ (presses $/ \mathrm{min}$ ), and rats that received Sal-ZIP at a rate of $13.4 \pm 2.18$.

In summary, these data are consistent with the hypothesis that ZIP altered the rats' ability to adapt to contingency shifts (see Fig. 5) by disrupting the contribution of PKM $\zeta$ to the normal trafficking of GluA2-containing AMPA receptors. This is because the effect of ZIP was blocked by an infusion of GluR2 $2_{3 Y}$, a peptide that prevents the normal endocytosis of GluA2-containing AMPA receptors, thus obviating the need for replacement that depends on PKM $\zeta$.

\section{Discussion}

These experiments support the currently accepted hypothesis that the dorsal medial and dorsal lateral striatal regions provide differential support for instrumental behavior (e.g., Yin et al. 2004, 2005a,b, 2006; Shiflett and Balleine 2011). Altering DLS function by infusing the NMDA antagonist DL-AP5 facilitated the rat's adaptation to a change in the reinforcement contingencies, whereas infusing it into the DMS impaired this adjustment. More importantly, our results support the hypothesis that these regions store task-relevant information and do not just coordinate the learning and retention of these solutions by other regions of the relevant neural system. This conclusion is based on the observation that inhibiting the putative memory-maintenance kinase PKM $\zeta$ following training on a biased concurrent reinforcement produced predictable and opposite effects when these contingencies were reversed. Specifically, infusing ZIP into the DLS facilitated performance during a contingency shift session. Presumably, this is because it erased memory content that supports previous learned habitual responding that would interfere with new required new learning and performance. In contrast, infusing ZIP into the DMS produced the impaired performance because it erased the previously acquired task information needed to assess and adapt to the contingency change.

A number of studies support the hypothesis that PKM $\zeta$ plays an essential role in the maintenance of potentiated synapses and in maintaining established memories (Pastalkova et al. 2006; Shema et al. 2007, 2011; Serrano et al. 2008; Hardt et al. 2010; $\mathrm{Li}$ et al. 2011). Moreover, data indicate that $\mathrm{PKM} \zeta$ is critical to regulating the trafficking of GluA2-containing AMPA receptors into the synapse that are needed to maintain strengthened synapses (Migues et al. 2010; Li et al. 2011). Nevertheless, concerns have been expressed that memory erasure with very high concentrations of ZIP, 100 times higher than the concentrations used in our experiments, may be caused by nonspecific effects other than the inhibition of PKM $\zeta$ (Lismann 2012).

Our results are novel in two ways. First, we used the PKM $\zeta$ methodology as an analytic tool to answer an important question-do the DMS and DLS areas store information that is critical for both assessing and adapting to contingency changes; do they store memories? Our results say the answer is yes. Second, the specific outcomes revealed that the two regions extract quite different content from the behavioral experience.

Our experiments support the view that the DMS and DLS support task-relevant memories. However, they in no way rule

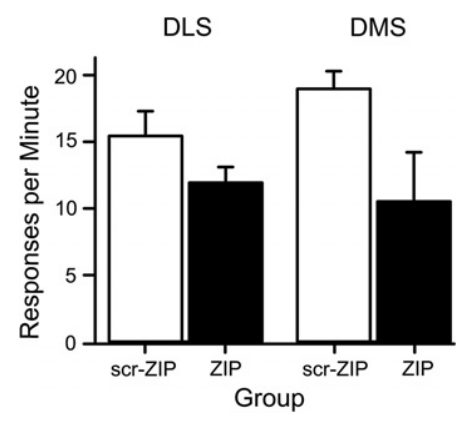

Figure 7. Mean number of responses per minute in the contingency shift session. Relative to scr-ZIP infusions, ZIP infusions reduced the leverpress rate independent of whether ZIP was infused into the DMS or DLS. 


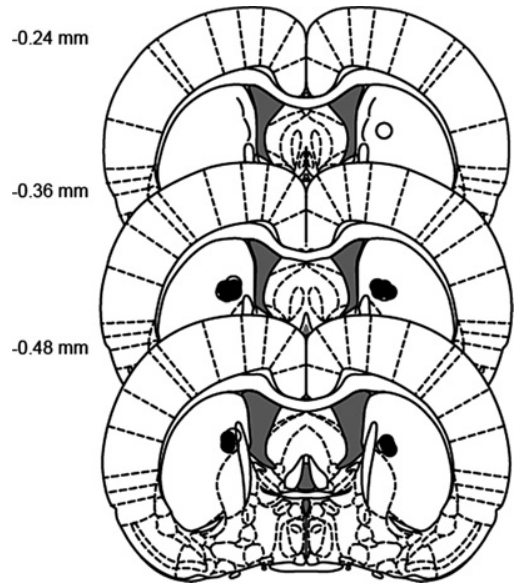

Figure 8. Schematic representation of cannulae tip placements for rats that received infusions into the DLS and DMS. Filled circles represent subjects that received GluR23Y and ZIP infusions, open circles represent subjects that received saline and ZIP infusions. Adapted from Paxinos and Watson (2008), with permission from Elsevier (c) 2008.

out the possibility that other brain structures, in particular, associated cortical regions, also store task-relevant information. Future experiments using the PKM $\zeta$ methodology will be needed to determine the involvement of other brain regions in tasks like ours.

Our experiments are also consistent with the current view that $\mathrm{PKM} \zeta$ maintains memories by regulating GluA2-containing AMPA receptor trafficking. This is because inhibition of endocytosis of these receptors by GluR2 $2_{3 Y}$ blocked the effect of ZIP. Our results, of course, are not definitive because we did not directly measure the location of these receptors. However, in the context of the existing literature, this hypothesis is reasonable.

Our data do not allow any definite conclusion about the content that is stored in the DMS or the DLS. However, the available data suggest that that the DLS stores stimulus-response associations (Reading et al. 1991; Packard and McGaugh 1996; Devan et al. 1999; Jog et al. 1999; Packard 1999; Featherstone and McDonald 2004; Yin et al. 2004; Palencia and Ragozzino 2005).

It is more difficult to describe the content of the DMS. Broadly speaking, some theorists assume that the DMS interacts with frontal brain regions to support a range of important functions such as working memory (Hazy et al. 2007), performance monitoring (Botvinick et al. 2001), and effort calculation (Walton et al. 2003). This suggests that DMS must learn to calculate the values of response alternatives based on the inputs it receives from these frontal areas (e.g., O'Doherty et al. 2004; Daw et al. 2005; Samejima et al. 2005). In the context of the present results, one might speculate that the DMS stores content needed to monitor ongoing response contingencies and detect changes in the these contingencies. In this way, it may contribute to rapid behavioral adjustments to contingency changes (Gallistel et al. 2001).

In conclusion, our results support the emerging hypothesis that two regions of the striatum, the DMS and DLS, make different contributions to instrumental behavior and to the rat's ability to adapt to change. More importantly, by suppressing the activity of the memory maintenance protein, $\mathrm{PKM} \zeta$, we provided the first evidence that these regions do not simply enable storage structures outside of the striatum to influence behavior but instead directly store information relevant to both behaving appropriately and adjusting to new contingencies.

\section{Materials and Methods}

\section{Animals}

Male Long-Evans rats (Harlan, Indianapolis, IN) served as subjects. A total of 49 rats, weighing between 325 and $349 \mathrm{~g}$ at the beginning of the study served as subjects. Two rats were excluded from analysis because of cannulae misplacement, three rats never learned to press levers, and one rat did not survive surgery. In the first study, five animals were implanted with DMS cannulae and five with DLS cannulae. This study was a within-subjects design: Each rat received DL-AP5 infusion in the first test cycle and vehicle saline infusions in the second test cycle. In the second study, which was not a within-subjects design, animals either received ZIP $(N:$ DMS $=6, \mathrm{DLS}=6)$ or scr-ZIP group $(N$ : DMS $=4, \mathrm{DLS}=$ $4)$. In the third study, rats received either saline infusions, followed by ZIP $1 \mathrm{~h}$ later $(N=6)$, or received GluR23y $(N=7)$, followed by ZIP $1 \mathrm{~h}$ later. Rats were housed individually in plastic cages in a temperature-controlled room, with free access to food and water for the first week. The vivarium maintained a 12-h lightdark cycle (lights on at 7:00 a.m.). Throughout the experiment, rats were food-restricted to maintain their weight at $\sim 90 \%$ of their ad libitum weight, with free access to water.

\section{Behavioral apparatus}

Training and testing took place in eight operant chambers equipped with two response levers (Coulbourn Instruments), placed $5 \mathrm{~cm}$ to the left and right of a liquid dipper (Coulbourn Instruments) that delivered $2 \%$ reduced-fat chocolate milk (Lucerne Foods) as reward. Each chamber was housed within a sound- and light-resistant cabinet. A 3-W, 5-V house-light, mounted in the center of the ceiling provided illumination. Experimental protocols were reviewed and approved by the University of Colorado Institutional Animal Care and Use Committee (IACUC).

\section{Behavioral procedures}

At the beginning of the experiment, a cup containing $15 \mathrm{~mL}$ of chocolate milk was placed in home cages for up to $2 \mathrm{~h}$. This procedure was repeated daily until subjects had consumed all of the chocolate milk within 20 min.

Rats then received daily lever-press training sessions that lasted $45 \mathrm{~min}$. They were trained on a variable temporal interval (VI) schedule of reinforcement. For each lever, the VI followed a uniform distribution. During the early stage of training, all rats developed a response bias that required special training to ensure that they pressed the two levers at approximately the same rate. For this purpose, a correction term was added to the VI of each

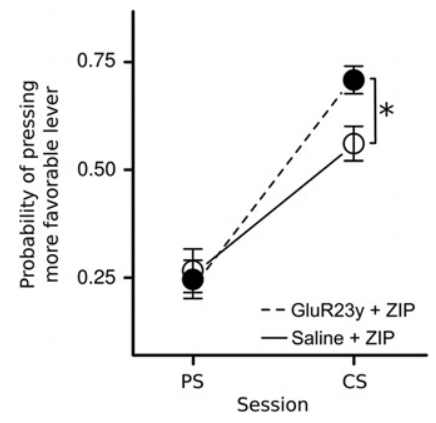

Figure 9. Probability of pressing the lever that was associated with the favorable VI $10^{\prime \prime}$ during the reversal session. On the day before the reversal session, when this lever was associated with the $\mathrm{VI} 40^{\prime \prime}$, rats in both groups showed a similar low probability of pressing this lever. If saline was infused into the DMS $1 \mathrm{~h}$ before ZIP, rats showed a decreased ability to adapt to the shift. If $\mathrm{GluR} 2_{3 Y}$ was infused into the DMS $1 \mathrm{~h}$ before ZIP, rats did show an adaptation to the contingency reversal that was similar to the control groups in the previous experiment that received infusions of scr-ZIP. Error bars indicate standard error (SE); $\left({ }^{*}\right) P<0.05$. 
lever to adjust for possible lever preferences: If a subject pressed one lever more often than the other, the difference in total leverpresses, since the beginning of the experiment, of this lever vs. the other lever was added (in sec) to the variable interval. This increased both the minimum and the maximum of the uniform distribution. On the first day of training, or until a rat had pressed each lever at least 50 times (since the beginning of the experiment), reward was delivered at a VI 0.5" (min: 0 sec, max: $1 \mathrm{sec}$ ), followed by one session during which reward was delivered at a VI $5^{\prime \prime}$ schedule (min: $0 \mathrm{sec}$, max: $10 \mathrm{sec}$ ). On the following days, rats were trained on a VI $20^{\prime \prime}$ schedule, until they pressed each lever at least 100 times per session and pressed both levers about equally often (fewer than 50 lever-presses difference between left and right lever). After rats met the training criteria, the cannulae were implanted, and rats were allowed $7 \mathrm{~d}$ to recover from surgery.

In the first experiment, in which DL-AP5 was injected prior to the contingency shift, rats were trained on concurrent VI $20^{\prime \prime}$ schedules for $2 \mathrm{~d}$ with the correction term still operative. This was followed by $2 \mathrm{~d}$ of training on the same schedule with the correction term removed.

Rats were then put through two 2-d test cycles. On day 1 of each test cycle, a VI $20^{\prime \prime}$ schedule was associated with each lever. On day 2 of cycles 1 and 2, the schedules were shifted: a less favorable VI $40^{\prime \prime}$ schedule was associated with the rat's preferred lever, and a more favorable VI 10" schedule was associated with the other lever. DL-AP5 was administered on day 2 of the first test cycle, and vehicle was injected on day 2 of the second test cycle. Thus, each rat was tested after a DL-AP5 infusion and after an infusion of the vehicle. After the first contingency shift on day 2 of cycle 1 , rats were retrained with concurrent VI $20^{\prime \prime}$ for $6 \mathrm{~d}$, before contingencies were shifted again on day 2 of test cycle 2 .

In the second and third experiment, in which PKM $\zeta$ was infused, rats were trained on an additional set of concurrent variable interval schedules. On the first day after post-surgical recovery, rats were again trained on concurrent VI $20^{\prime \prime}$ schedules without a correction term to determine lever preference. For the following sessions, the lever a rat preferred during this session was assigned a less favorable VI $40^{\prime \prime}$ schedule and the other lever a more favorable VI $10^{\prime \prime}$ schedule. Rats were trained according to this schedule for six sessions. Following the completion of the last bias training session, the rats in the DMS and DLS conditions were permuted such that they received either ZIP or scr-ZIP. Thus, during reversal, rats were returned to their original bias. A similar strategy has been applied before by others to investigate the role of the two dorsal striatal areas in behavioral flexibility (e.g., Palencia and Ragozzino 2005).

In experiment 2, either ZIP or scr-ZIP was infused into the relevant region $\sim 24 \mathrm{~h}$ after the last session of bias training. To determine if ZIP influenced memory maintenance, $48 \mathrm{~h}$ later, rats were returned to the operant chambers and exposed to a contingency shift-the lever-VI schedule assignments during bias training were reversed during the test session.

The procedures in experiment 3 were identical to the procedures in experiment 2, except for the drug infusions. Rats were permuted again and received either saline infusions, followed by ZIP $1 \mathrm{~h}$ later, or received GluR23y, followed by ZIP $1 \mathrm{~h}$ later.

\section{Surgery}

Rats were anesthetized with Halothane and stereotaxically implanted with 26-gauge stainless steel guide cannulae (Plastics One) bilaterally into the posterior dorsomedial or dorsolateral striatum. Bregma was used as a reference. We used the following coordinates for bilateral implantation in the dorsomedial striatum: anteriorposterior, $-0.4 \mathrm{~mm}$; mediolateral, $\pm 2.6 \mathrm{~mm}$; dorsoventral, $4.5 \mathrm{~mm}$. We used the following coordinates for bilateral implantation in the dorsolateral striatum: anteriorposterior, +0.7 $\mathrm{mm}$; mediolateral, $\pm 3.6 \mathrm{~mm}$; dorsoventral, $5.0 \mathrm{~mm}$.

\section{Microinfusions}

We gently wrapped the rat in a soft towel, removed the obturator, and inserted a 33-gauge microinjector (Plastics One) attached to polyethylene 50 (PE50) tubing through the indwelling guide cannula. The distal end of the PE50 tubing was attached to a $10-\mathrm{mL}$ (Hamilton) syringe that was attached to a microinjection unit (model 5000; David Kopf Instruments) that accurately dispensed the desired volume at a rate of $0.5 \mu \mathrm{L} / \mathrm{min}$. The microinjectors extended $0.5 \mathrm{~mm}(1 \mathrm{~mm}$ in experiment 1$)$ into striatum beyond the tip of the guide cannulae. The injector remained connected for an additional minute to allow for drug to diffuse away from the tip of the cannula.

\section{Drugs}

In the first experiment, DL-AP5, a selective NMDA antagonist provided by Tocris in powder form, was dissolved in a saline solution. We used a concentration of $4.5 \mu \mathrm{g} / \mu \mathrm{L}$, injecting $0.5 \mu \mathrm{L}$ in $1 \mathrm{~min}$ for a total of $2.25 \mu \mathrm{g}$ per side. Saline vehicle injections with the same parameters were used as control. We chose this drug for two reasons. First, the primary projection neurons in the striatum are the medium spiny neurons, and there is evidence that NMDA receptors modulate their excitability. These neurons alternate between a hyperpolarized resting membrane potential ("down state") and transient periods of a sustained depolarization ("up state") (Wickens and Wilson 1998), during which MSNs show an increased responsiveness to synaptic input. Antagonism of D1 dopamine and AMPA glutamatergic receptors have been found to block transitions into up states (Surmeier et al. 2007; Tseng et al. 2007), and NMDA receptor antagonism with AP5 has been reported to significantly shorten the duration of up states (Tseng et al. 2007). This shortened duration associated with antagonizing NMDA receptors might mediate the effects produced when DLAP5 was injected into the striatum. Second, DL-AP5 has relatively minor effects on overall instrumental performance, unlike other drugs (e.g., muscimol) that greatly impact behavioral function when injected into the striatum (Kelley et al. 2003).

In the second experiment, we dissolved either the PKM $\zeta$ inhibitor ZIP (30 nmol/side/0.5 $\mu \mathrm{L}$; Myr-SIYRRGARRWRKL; AnaSpec, catalog \#63361) or an inactive scrambled version of the peptide (scr-ZIP; $30 \mathrm{nmol} /$ side/0.5 $\mu \mathrm{L}$; Myr-RLYRKRIWRSAGR; Anaspec, catalog \#63695) in saline (pH 7.0), and injected it into the DMS or the DLS.

In the third experiment, we first injected either Tat-GluR2 $2_{3 Y}$ (45 pmol/side/0.5mul; YGRKKRRQRRRYKEGYNVYG; Anaspec, catalog \#64429) dissolved in saline ( $\mathrm{pH} 7.0$ ) or vehicle. One h later, we then injected the PKM $\zeta$ inhibitor ZIP (30 nmol/side $/ 0.5 \mu \mathrm{L}$; Myr-SIYRRGARRWRKL; AnaSpec, catalog \#63361).

\section{Histology}

At the completion of the experiment, we anesthetized rats with pentobarbital (50 mg per kg body weight), decapitated them, removed their brains, and froze the brains in cold isopentane. We cut coronal sections ( $40-\mu \mathrm{m}$ thick) through the striatum with a cryostat and mounted every third section. We stained sections with cresyl violet and examined them by light microscopy to visually verify the placement of the cannulae. Only rats with proper cannula placements were included in the experiment.

\section{Statistics}

If the time between the current lever-press and the previous response was larger than the median response interval between lever-presses plus three times the standard deviation, the current lever-press was removed from analysis. To measure how well rats adapted to a contingency change, we calculated the relative probability of pressing the lever associated with the more favorable schedule. Specifically, we divided the number of presses of the richer lever by the total number of lever presses.

For the purpose of determining how well a rat had adapted to new contingencies, we compared the relative probability of pressing a lever in the second half of the contingency shift session with the same relative probability during the second half of the last session before the contingency shift. Specifically, we determined for each rat the total number of lever-presses in a session and included 
the last $50 \%$ of those lever-presses in our analysis. To determine the time-course of adaptation, we calculated how the relative probability of pressing the more favorable lever changed with progress through a session.

For statistical support we used a strictly hierarchical approach, using repeated-measures analysis of variance, followed by planned $t$-test comparisons to explain found interactions (using the statistics program R). $T$-test results are only reported where underlying interaction effects were significant in the corresponding repeated measures ANOVAs. Values were rounded to the second decimal.

\section{Acknowledgments}

We thank Dr. Timothy Curran, Dr. Matt Jones, Dr. Christopher Lowry, and members of the Computational Cognitive Neuroscience Laboratory at the University of Colorado at Boulder for constructive discussions, and Brittany Thompson, Dr. Rob Rozeske II, Dr. John Christianson, Kyle Rolofson, and Matthew Pomrenze for help with data collection.

\section{References}

Adams CD, Dickinson A. 1981. Instrumental responding following reinforcer devaluation. Q J Exp Psychol B 33: 109-121.

Botvinick M, Braver TS, Barch DM, Carter CS, Cohen JD. 2001. Conflict monitoring and cognitive control. Psychol Rev 108: 624-652.

Daw ND, Niv Y, Dayan P. 2005. Uncertainty-based competition between prefrontal and dorsolateral striatal systems for behavioral control. Nat Neurosci 8: 1704-1711.

Devan BD, McDonald RJ, White NM. 1999. Effects of medial and lateral caudate-putamen lesions on place- and cue-guided behaviors in the water maze: Relation to thigmotaxis. Behav Brain Res 100: 5-14.

Dickinson A. 1980. Contemporary animal learning theory. Cambridge University Press, Cambridge, UK.

Dickinson A, Balleine B. 1993. Actions and responses: The dual psychology of behaviour. In Spatial representation (ed. N Eilan, et al.), pp. 277-293. Blackwell Publishers, Cambridge, MA.

Everitt BJ, Robbins TW. 2005. Neural systems of reinforcement for drug addiction: From actions to habits to compulsion. Nat Neurosci 8: 1481-1489.

Featherstone RE, McDonald RJ. 2004. Dorsal striatum and stimulusresponse learning: Lesions of the dorsolateral, but not dorsomedial, striatum impair acquisition of a simple discrimination task. Behav Brain Res 150: 15-23.

Gallistel CR, Mark TA, King AP, Latham PE. 2001. The rat approximates an ideal detector of changes in rates of reward: Implications for the law of effect. J Exp Psychol Anim Behav Process 27: 354-372.

Hardt O, Migues PV, Hastings M, Wong J, Nader K. 2010. PKM $\zeta$ maintains 1-day- and 6-day-old long-term object location but not object identity memory in dorsal hippocampus. Hippocampus 20: 691-695.

Hazy TE, Frank MJ, O'Reilly RC. 2007. Towards an executive without a homunculus: Computational models of the prefrontal cortex/basal ganglia system. Philos Trans R Soc Lond B Biol Sci 362: 1601-1613.

Hilário MRF, Costa RM. 2008. High on habits. Front Neurosci 2: 208-217.

Jog MS, Kubota Y, Connolly CI, Hillegaart V, Graybiel AM. 1999. Building neural representations of habits. Science 286: 1745-1749.

Kelley AE, Andrzejewski ME, Baldwin AE, Hernandez PJ, Pratt WE. 2003 Glutamate-mediated plasticity in corticostriatal networks: Role in adaptive motor learning. Ann N Y Acad Sci 1003: 159-168.

Killcross S, Coutureau E. 2003. Coordination of actions and habits in the medial prefrontal cortex of rats. Cereb Cortex 13: 400-408.

Li Y, Xue Y, He Y, Li F, Xue L, Xu C, Sacktor TC, Shaham Y, Lu L. 2011. Inhibition of PKM $\zeta$ in nucleus accumbens core abolishes long-term drug reward memory. J Neurosci 31: 5436-5446.

Lisman J. 2012. Memory erasure by very high concentrations of ZIP may not be due to PKM- $\zeta$. Hippocampus 22: 648-649.

Migues PV, Hardt O, Wu DC, Gamache K, Sacktor TC, Wang YT, Nader K. 2010. PKM $\zeta$ maintains memories by regulating GluR2-dependent AMPA receptor trafficking. Nat Neurosci 13: 630-634.

O'Doherty J, Dayan P, Schultz J, Deichmann R, Friston K, Dolan RJ. 2004. Dissociable roles of ventral and dorsal striatum in instrumental conditioning. Science 304: 452-454.

Packard MG. 1999. Glutamate infused posttraining into the hippocampus or caudate-putamen differentially strengthens place and response learning. Proc Natl Acad Sci 96: 12881-12886.
Packard MG, McGaugh JL. 1996. Inactivation of hippocampus or caudate nucleus with lidocaine differentially affects expression of place and response learning. Neurobiol Learn Mem 65: 65-72.

Palencia CA, Ragozzino ME. 2005. The contribution of NMDA receptors in the dorsolateral striatum to egocentric response learning. Behav Neurosci 119: 953-960.

Pastalkova E, Serrano P, Pinkhasova D, Wallace E, Fenton AA, Sacktor TC. 2006. Storage of spatial information by the maintenance mechanism of LTP. Science 313: 1141-1144.

Pasupathy A, Miller EK. 2005. Different time courses for learningrelated activity in the prefrontal cortex and striatum. Nature $\mathbf{4 3 3}$ : $873-876$.

Pauli WM, Atallah HE, O'Reilly RC. 2010. Integrating what \& how/where with instrumental and Pavlovian learning: A biologically based computational model. In Cognition and neuropsychology: International perspectives on psychological science (ed. PA Frensch, R Schwarzer), Vol. 1, pp. 71-95. Psychology Press, East Sussex, UK.

Pauli W, Hazy T, O’Reilly R. 2012. Expectancy, ambiguity, and behavioral flexibility: Separable and complementary roles of the orbital frontal cortex and amygdala in processing reward expectancies. J Cogn Neurosci 24: $351-366$.

Paxinos G, Watson C. 2008. The rat brain in stereotaxic coordinates, 6th Ed. Academic Press, Waltham, MA.

Ragozzino ME. 2007. The contribution of the medial prefrontal cortex, orbitofrontal cortex, and dorsomedial striatum to behavioral flexibility. Ann N Y Acad Sci 1121: 355-375.

Reading PJ, Dunnett SB, Robbins TW. 1991. Dissociable roles of the ventral, medial and lateral striatum on the acquisition of a complex visual stimulus-response habit. Behav Brain Res 45: 147-161.

Rudy J. 2008. The neurobiology of learning and memory. Sinauer Associates, Inc., Sunderland, MA.

Sacktor TC. 2011. How does PKM $\zeta$ maintain long-term memory? Nat Rev 12: $9-15$.

Samejima K, Ueda Y, Doya K, Kimura M. 2005. Representation of actionspecific reward values in the striatum. Science 310: 1337-1340.

Serrano P, Yao Y, Sacktor TC. 2005. Persistent phosphorylation by protein kinase M $\zeta$ maintains late-phase long-term potentiation. J Neurosci 25: 1979-1984.

Serrano P, Friedman EL, Kenney J, Taubenfeld SM, Zimmerman JM, Hanna J, Alberini C, Kelley AE, Maren S, Rudy JW, et al. 2008. PKM $\zeta$ maintains spatial, instrumental, and classically conditioned long-term memories. PLOS Biol 6: 2698-2706.

Shema R, Sacktor TC, Dudai Y. 2007. Rapid erasure of long-term memory associations in the cortex by an inhibitor of PKM $\zeta$. Science 317: 951-953.

Shema R, Haramati S, Ron S, Hazvi S, Chen A, Sacktor TC, Dudai Y. 2011. Enhancement of consolidated long-term memory by overexpression of protein kinase $\mathrm{M} \zeta$ in the neocortex. Science 331: 1207-1210.

Shiflett MW, Balleine BW. 2011. Molecular substrates of action control in cortico-striatal circuits. Prog Neurobiol 99: 1-55.

Surmeier DJ, Ding J, Day M, Wang Z, Shen W. 2007. D1 and D2 dopamine-receptor modulation of striatal glutamatergic signaling in striatal medium spiny neurons. Trends Neurosci 30: 228-235.

Thorndike EL. 1898. Animal intelligence: An experimental study of associative processes in animals. Psychol Monogr 2: (Suppl No 4) 1-109.

Tolman E. 1948. Cognitive maps in rats and men. Psychol Rev 55: 189-208.

Tseng KY, Snyder-Keller A, O'Donnell P. 2007. Dopaminergic modulation of striatal plateau depolarizations in corticostriatal organotypic cocultures. Psychopharmacology 191: 627-640.

Walton ME, Bannerman DM, Alterescu K, Rushworth MF. 2003. Functional specialization within medial frontal cortex of the anterior cingulate for evaluating effort-related decisions. J Neurosci 23: 6475-6479.

Wickens JR, Wilson CJ. 1998. Regulation of action-potential firing in spiny neurons of the rat neostriatum in vivo. J Neurophysiol 79: 2358-2364.

Yin HH, Knowlton BJ, Balleine BW. 2004. Lesions of dorsolateral striatum preserve outcome expectancy but disrupt habit formation in instrumental learning. Eur J Neurosci 19: 181-189.

Yin HH, Knowlton BJ, Balleine BW. 2005a. Blockade of NMDA receptors in the dorsomedial striatum prevents action-outcome learning in instrumental conditioning. Eur J Neurosci 22: 505-512.

Yin HH, Ostlund SB, Knowlton BJ, Balleine BW. 2005b. The role of the dorsomedial striatum in instrumental conditioning. Eur J Neurosci 22: $513-523$.

Yin HH, Knowlton BJ, Balleine BW. 2006. Inactivation of dorsolateral striatum enhances sensitivity to changes in the action-outcome contingency in instrumental conditioning. Behav Brain Res 166: 189-196.

Received December 1, 2011; accepted in revised form April 2, 2012. 


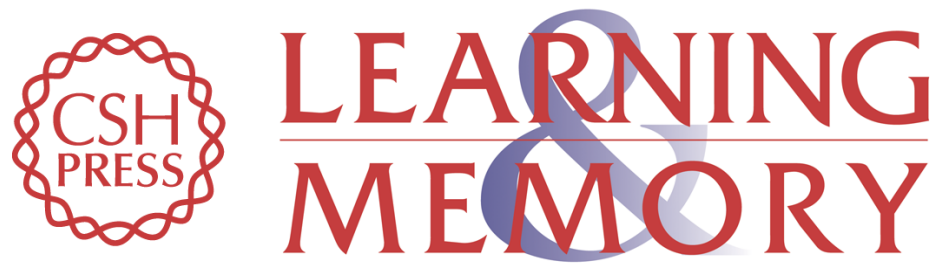

\section{Inhibiting PKM $\zeta$ reveals dorsal lateral and dorsal medial striatum store the different memories needed to support adaptive behavior}

Wolfgang M. Pauli, Alexandra D. Clark, Heidi J. Guenther, et al.

Learn. Mem. 2012, 19:

Access the most recent version at doi:10.1101//m.025148.111

References This article cites 43 articles, 10 of which can be accessed free at:

http://learnmem.cshlp.org/content/19/7/307.full.html\#ref-list-1

License

Email Alerting Receive free email alerts when new articles cite this article - sign up in the box at the Service top right corner of the article or click here. 